\title{
Evolución y estructura de la población en situación de dependencia
}

\author{
Antonio ABELLÁN GARCÍA \\ Consejo Superior de Investigaciones Científicas \\ Antonio.abellan@cchs.csic.es \\ Cecilia EsPARZA CATALÁN \\ Consejo Superior de Investigaciones Científicas \\ Cecilia.esparza@cchs.csis.es \\ Julio PÉREZ DÍAZ \\ Consejo Superior de Investigaciones Científicas \\ Julio.perez@cchs.csic.es
}

\begin{abstract}
RESUMEN
La dependencia se define por la incapacidad funcional en actividades de la vida diaria y por requerir ayuda para realizarlas. En España hay dos millones de personas dependientes (2008). Se hace más probable con la edad y es superior entre las mujeres y las personas de menor nivel de instrucción. Existe una relación entre el orden de aparición y la prevalencia de las discapacidades que pueden provocar dependencia: los problemas de movilidad exterior suelen ser los primeros y los más frecuentes; le siguen las dificultades para tareas de hogar y se finaliza con los problemas de cuidado personal. La ayuda familiar es la principal respuesta; la mujer desempeña un papel fundamental como madre de joven dependiente, madre, esposa o hija de varón adulto con discapacidad, o hija de padres mayores con dificultades funcionales.
\end{abstract}

Palabras clave: Dependencia, actividades de la vida diaria, medida, características sociodemográficas, severidad, cuidados familiares, ayuda informal.

\section{Evolution and structure of dependent people}

\begin{abstract}
Dependency is defined for the purpose of this paper as having difficulties for activities of daily living and the need of receiving personal care. There are two millions of dependent people in Spain (2008). Dependency rate increase with age and are higher among women and people of lower educational level. There is a relationship between the sequence order and the prevalence of disabilities that lead to dependency. Outdoor mobility problems are usually the first and most commonly cited problems, followed by difficulties in performing housework and subsequently by difficulties in basic activities of personal care. Family support is the most common answer to dependency. Women play a key role: as mothers of young dependents, mothers, wives or daughter of adult males with disabilities, or daughters of parents with functional disabilities.
\end{abstract}

Keywords: dependency, activities of daily living, measures, socio-demographic characteristics, severity, family caregivers, informal care.

\section{REFERENCIA NORMALIZADA}

Abellán García, A., Esparza Catalá, C. y Pérez Díaz J. (2011) Evolución y estructura de la población en situación de dependencia. Cuadernos de Relaciones Laborales Vol. 29, núm. 1, p. 43-67. 
SUMARIO: 1 Introducción. 2. Material y procedimiento para el cálculo de la dependencia. 3. Las grandes cifras de la dependencia funcional. 4. Variaciones por sexo y edad. 5. Otras características sociodemográficas. 6. Prevalencia de las limitaciones en las actividades de la vida diaria. 7. Principales enfermedades asociadas a la dependencia. 8. Severidad. 9. La recepción de ayuda. 10. Conclusiones. Bibliografía.

\section{Introducción}

El objetivo de esta breve colaboración es hacer una síntesis de las principales características de la población española en situación de dependencia. Pero para ello primero es necesario saber cómo se define y cómo se mide.

Existen diferentes maneras de hacerlo; por su naturaleza la dependencia puede ser física, psicológica, emocional, económica, etc. También sus causas pueden ser diversas: accidente o crisis, estadio del ciclo de vida, salud, personalidad o expectativas sociales, además de las discapacidades funcionales (Wilkin, 1987).

Es importante distinguir aquí entre discapacidad y dependencia:

- Discapacidad.- Es la dificultad para llevar a cabo actividades de la vida cotidiana, habituales para personas de similar edad y condición sociocultural. Por tanto incluye dos polos, las capacidades personales y las demandas del entorno respecto a las actividades a realizar normalmente (Spector y Fleishman, 1998; Puga y Abellán, 2004; Querejeta, 2004; Abellán y Esparza, 2007). La dificultad para realizar esas actividades (poca, moderada, severa o absoluta) califica a una persona con discapacidad.

- Dependencia.- Es la situación de una persona con discapacidad que solicita ayuda de otra para esas actividades cotidianas porque no puede valerse por sí misma.

Nuestro criterio definitorio va a ser la capacidad de desempeñarse autónomamente en tres ámbitos fundamentales de la vida cotidiana: el autocuidado personal, la gestión del hogar y la relación con la comunidad y el entorno. Y es sobre todo la dependencia física, causada mayoritariamente por discapacidades funcionales, la que causa las demandas más básicas de ayuda en estos ámbitos.

Nos centramos por tanto en este tipo, la dependencia física, adoptando como medida para estudiarlo el funcionamiento físico en actividades básicas, instrumentales y de movilidad (Börsch-Supan, 2005). Se busca así conocer las capacidades físicas del individuo para manejarse y tratar de mantener su autonomía personal, y contabilizar las personas que tienen problemas y necesitan ayuda personal para realizarlas (Rodríguez Cabrero, 2004).

Situaciones de dependencia.- En función de la necesidad de ayuda, su recepción o no, y el grado de satisfacción con la que se recibe, deben hacerse algunas distinciones importantes (Gignac y Cott, 1998). "Persona dependiente" es la que por una discapacidad necesita ayuda para desarrollar actividades cotidianas, y la recibe. Si la ayuda satisface sus necesidades, se considera una demanda cubierta, pero a veces no es así, la ayuda es "insuficiente" y se demanda atención adicional. 
Puede ocurrir también que se reciba asistencia que no se considere necesaria. Se produce entonces una "dependencia impuesta" (por sobreprotección, por "prevención" u otras actitudes que a veces resultan de cambios relacionales como la reagrupación familiar o el fallecimiento de un cónyuge).

Si alguien con discapacidad no recibe ni pide ayuda, es una persona "independiente", como lo son todas las que no tienen problemas funcionales. Pero si la pide y no la recibe la incluimos entre los dependientes ("dependiente potencial con demanda insatisfecha", cosa que suele relacionarse con circunstancias familiares adversas, o con el desconocimiento de los servicios al alcance).

En los centros y alojamientos colectivos (residencias, hospitales geriátricos, centros para personas con discapacidad, etc.) se entiende que todas las personas con alguna limitación funcional reciben atención para actividades cotidianas (instrumentales, al menos), y en algún caso para actividades básicas, lo que las incluye automáticamente bajo el concepto de dependiente.

En resumen, dependiente es la persona con discapacidad que recibe ayuda (suficiente, insuficiente o innecesaria) o que la solicita pero no la recibe.

\section{Material y procedimiento para el cálculo de la dependencia}

Datos.- Se han utilizado los microdatos de la Encuesta sobre Discapacidad, Autonomía personal y situaciones de Dependencia (EDAD), de 2008. Es la tercera gran operación estadística del INE sobre discapacidad. Sus antecedentes son la Encuesta sobre Discapacidades, Deficiencias y Minusvalías de 1986, y la Encuesta sobre Discapacidades, Deficiencias y Estado de Salud (EDDES) de 1999. A esta última se hacen referencias puntuales para establecer la evolución de la dependencia en los últimos nueve años.

EDAD busca estimar el número de personas con discapacidad, conocer el tipo de limitación, las características de las personas en situación de dependencia e identificar necesidades y demandas de asistencia. El trabajo de campo se desarrolló entre noviembre de 2007 y febrero de 2008. Se recogió información de todas las personas de los hogares seleccionados ( $1^{\text {a }}$ fase) y en caso de existir alguna con discapacidad (6 y más años) o limitaciones (0-5 años) se pasó un cuestionario específico ( $2^{\mathrm{a}}$ fase); si esa persona recibía cuidados se completaba el cuestionario con un pequeño módulo de cuidadores principales. También se realizó la encuesta en centros e instituciones, una novedad respecto a las dos anteriores que supondrá, por primera vez, la posibilidad de estudiar la relación entre discapacidad y edades avanzadas sin la perturbación que hasta ahora suponía la salida de observación por ingreso en residencias colectivas ${ }^{1}$.

${ }^{1}$ La muestra de EDAD fue de 96.075 viviendas familiares; se recogió información en la primera fase de 258.187 personas; de esos registros, 22.795 correspondían a personas con discapacidad; en la segunda fase se recogió información del módulo específico de discapaci- 
Las dos primeras encuestas utilizaron el marco conceptual de la primera Clasificación Internacional de Deficiencias, Discapacidades y Minusvalías de la OMS, 1980 (Imserso, 1994). La actual encuesta EDAD se ha realizado tras haber aparecido la nueva Clasificación Internacional del Funcionamiento, de la Discapacidad y de la Salud (CIF) de 2001 (OMS, 2001)2.

Medida.- No existe un instrumento único o universalmente aceptado para calcular la prevalencia de la discapacidad y la dependencia en cada encuesta. Las diferencias en la definición, construcción y evaluación de las medidas, hacen difícilmente comparables sus resultados (Béland y Zunzunegui, 1999; Morán, 1999; Rodríguez Cabrero, 2004; Imserso, 2004; Querejeta, 2004; Puga y Abellán, 2004; Alegre et al., 2005; Albarrán y Alonso, 2006; Abellán y Esparza, 2007; Cabrero, 2007). Eso no obsta para que esa información pueda ser incorporada a estrategias de políticas públicas e intervención social.

La medida de capacidad funcional es muy útil respecto a los mayores, dada su estrecha relación con la edad. Para el resto de la población sus valores suelen ser muy bajos, por lo que conviene agregar la población en grandes grupos para analizarla. Se estimará la discapacidad y dependencia de las personas de seis y más años y se delimitarán tres grupos de edad: 6-19, 20-64 y 65 ó más años, que corresponden aproximadamente con las personas que se encuentra en el sistema educativo, las que pueden estar en el mercado laboral y las personas mayores, respectivamente.

Se acota el estudio a la población que reside en viviendas familiares y tiene 6 y más años. También se ha estimado la población dependiente que reside en centros e instituciones de alojamiento colectivo; en éstos no se puede especificar la edad nada más que en dos grandes grupos, siendo el de 65 y más años el preponderante.

dad (6 y más años) de 21.583 personas (1.212 personas con discapacidad no respondieron al módulo específico); un $32 \%$ de las respuestas de este módulo fueron cumplimentadas por individuos diferentes a la persona con discapacidad (informantes); además, se recogió información de 381 niños de 0-5 años con limitaciones, con su módulo específico. En los alojamientos residenciales colectivos se entrevistaron 10.567 personas de todas las edades.

${ }^{2}$ Los cambios más notables entre una y otra clasificación de la OMS (1980 y 2001) son en parte terminológicos: deficiencia se refiere ahora a funciones y estructuras corporales; discapacidad se cambia a funcionamiento (actividad y participación), es decir, en clave positiva; minusvalía desaparece del esquema; el modelo de la discapacidad y minusvalía es ahora del funcionamiento y de la discapacidad. Pero también hay otros cambios. Antes, discapacidad era cualquier restricción o falta de habilidad, consecuencia de una deficiencia; y ahora es un paraguas que abarca los problemas en las estructuras corporales, en sus funciones, y también las limitaciones en la actividad y restricciones en la participación (Whiteneck, 2005; Lafortune, 2007; OMS, 2001, p. 11; Abellán y Esparza, 2007). Se han incluido los factores ambientales y personales. Este punto de vista es fundamental cuando se considera que la discapacidad entra en el campo de lo social y no sólo en el médico (Abellán, Castejón, Esparza, 2007). Los términos actividad y participación, con una lista única de calificadores, no están suficientemente aclarados en la Clasificación de 2001. 
Para la finalidad de este artículo se han computado trece actividades que definen la capacidad y el funcionamiento de los individuos, y además son comparables con ítems casi idénticos de EDDES $1999^{3}$. Esas actividades se han agrupado en tres dominios:

a) cuidado personal, es decir, actividades básicas de autocuidado necesarias para mantener la independencia personal; incluye las tareas de levantarse/acostarse, vestirse, lavarse y cuidados básicos del cuerpo, control de necesidades (orinar, defecar), desplazarse dentro de la casa, control de prescripciones médicas, y comer y beber;

b) tareas domésticas, es decir, actividades instrumentales necesarias para mantener una vida independiente en el hogar; incluye preparar comidas y ocuparse de las tareas de la casa; y

c) movilidad exterior, engloba andar fuera y desplazarse utilizando medios de transporte, que se han diferenciado de las de desplazamiento dentro de la casa que habitualmente se incluyen como actividades básicas; realizar compras puede ser considerada dentro del dominio de la movilidad exterior, aunque también suele ser incluida en las meras actividades instrumentales.

Severidad.- Para su cálculo de la severidad se ha asignado un valor de "carga" a cada discapacidad en función de su naturaleza (agrupando discapacidades en tres grandes conjuntos mediante análisis factorial), su frecuencia de aparición y la propia declaración del individuo sobre el nivel de dificultad para la realización de las actividades cotidianas $^{4}$. El valor así obtenido abarca una escala continua de cero a uno. Se han

${ }^{3}$ Desgraciadamente los 44 ítems de EDAD no coinciden plenamente con los 36 de la EDDES. Sólo en 24 se preguntó específicamente si recibían atención personal. De forma genérica se preguntó (bloque L) si recibían asistencia o cuidados personales, en conjunto; pero ese filtro no pudo utilizarse dado que no se utilizaron todas las actividades, y podría darse el caso de tener discapacidad en un ítem, no recibir ayuda en esa actividad en concreto, pero haber respondido genéricamente que se recibían cuidados (en otras). Es decir, ese filtro de dependencia sólo es utilizable cuando el indicador de discapacidad incluye las 44 actividades, y esto es improcedente en el marco conceptual adoptado de incapacidad funcional.

${ }^{4}$ Pasos seguidos para el cálculo de la severidad:

a) Reducción de las dimensiones de las 13 actividades de la vida diaria consideradas mediante un análisis factorial. A partir de este análisis las actividades se agrupan en tres conjuntos (autocuidados, tareas domésticas y movilidad).

b) Se suman los valores de gravedad, declarados por los individuos, en las variables o ítems que conforman cada uno de esos conjuntos.

c) Las puntuaciones obtenidas en el paso B se modifican para que tomen valores entre cero y uno. 
agrupado las puntuaciones siguiendo la guía de calificadores de la CIF (OMS, 2001, p. 241): 0 a 0,04 , no hay problema o es insignificante; 0,05 a 0,24 , severidad leve; 0,25 a 0,49 , moderada; 0,50 a 1 , grave (incluyendo total o completa).

\section{Las grandes cifras de la dependencia funcional}

En España hay 2.141.404 personas dependientes de 6 y más años, residiendo en viviendas familiares, con diferente grado de severidad, según los criterios aquí propuestos $^{5}$; esa cifra representa un $5,1 \%$ de toda la población de 6 y más años, y ocho de cada diez personas con discapacidad (2.745.855). Otras 258.327 residen en centros y alojamientos colectivos (residencias, hospitales de larga estancia, geriátricos, psiquiátricos y centros residenciales para personas con discapacidad).

Los diferentes supuestos de dependencia se reparten de la siguiente forma: a) la inmensa mayoría (988.153 personas) tienen la demanda de ayuda cubierta y la asistencia satisface sus necesidades; b) existe casi medio millón de dependientes (474.922) con atención insuficiente; c) un 4\% de los dependientes estima que la ayuda que reciben es innecesaria, quizá por sobreprotección o por la forma de convivencia adoptada; d) además, hay 176.805 personas con discapacidad que no reciben asistencia pero la demandan; son personas "no independientes" computadas dentro del colectivo de dependientes. Las personas con discapacidad pero con independencia

d) Se asigna una ponderación a cada uno de los conjuntos basada en el principio de mayor peso cuanto menor es el número de individuos afectados. Para ello, en cada uno de los conjuntos se multiplican las puntuaciones obtenidas en el paso C por el número de individuos que las presentan. Se suma el resultado de cada una de esas multiplicaciones. El valor más pequeño de dicha suma en los tres conjuntos es tomado como referencia para el cálculo de las ponderaciones.

e) Se multiplica la puntuación de cada conjunto (obtenida en el paso C) por su correspondiente ponderación (obtenida en el paso D). El resultado de la variable indicadora de la severidad es la suma de dichos productos.

${ }^{5}$ Se ha calculado, a efectos comparativos, la dependencia que supondría considerar los 44 ítems de EDAD, aunque la mayoría de esos ítems no tengan cabida en la propuesta teórica que aquí se hace, centrada en los problemas funcionales (En EDAD se mezclan deficiencias, limitaciones funcionales y problemas en la realización de actividades de la vida diaria). Considerando todos, habría 3,8 millones de personas con discapacidad, y 2.327.459 personas dependientes, que reciben ayuda o que demandan sin recibirla; es decir, sólo un 8,6\% de dependientes más que en nuestro cálculo. En cambio, se estaría hablando de un millón adicional de personas con discapacidad, de una forma que en nuestra opinión distorsiona el escenario de la discapacidad y de la demanda de asistencia. 
funcional, pues pueden manejarse para actividades cotidianas, se elevan a $411.034 \mathrm{y}$ están fuera del cómputo (Figura 1).

En 1999, según la EDDES, había en España 1.621.909 personas dependientes, contabilizadas con el mismo procedimiento ${ }^{6}$; eran el $4,4 \%$ de la población de 6 y más años, que residía en viviendas familiares. Ahora, en 2008, hay más dependientes en cifras absolutas y su tasa es también superior.

${ }^{6}$ A pesar de utilizar el mismo procedimiento, las preguntas en 1999 no fueron idénticas para conocer la recepción de ayuda. La causa principal de esta diferencia estriba en los que no reciben pero demandan ayuda (clasificados como dependientes), que en 2008 eran 153.028 personas más que en 1999 . 
Figura 1.- Personas de 6 y más años dependientes. España, 2008

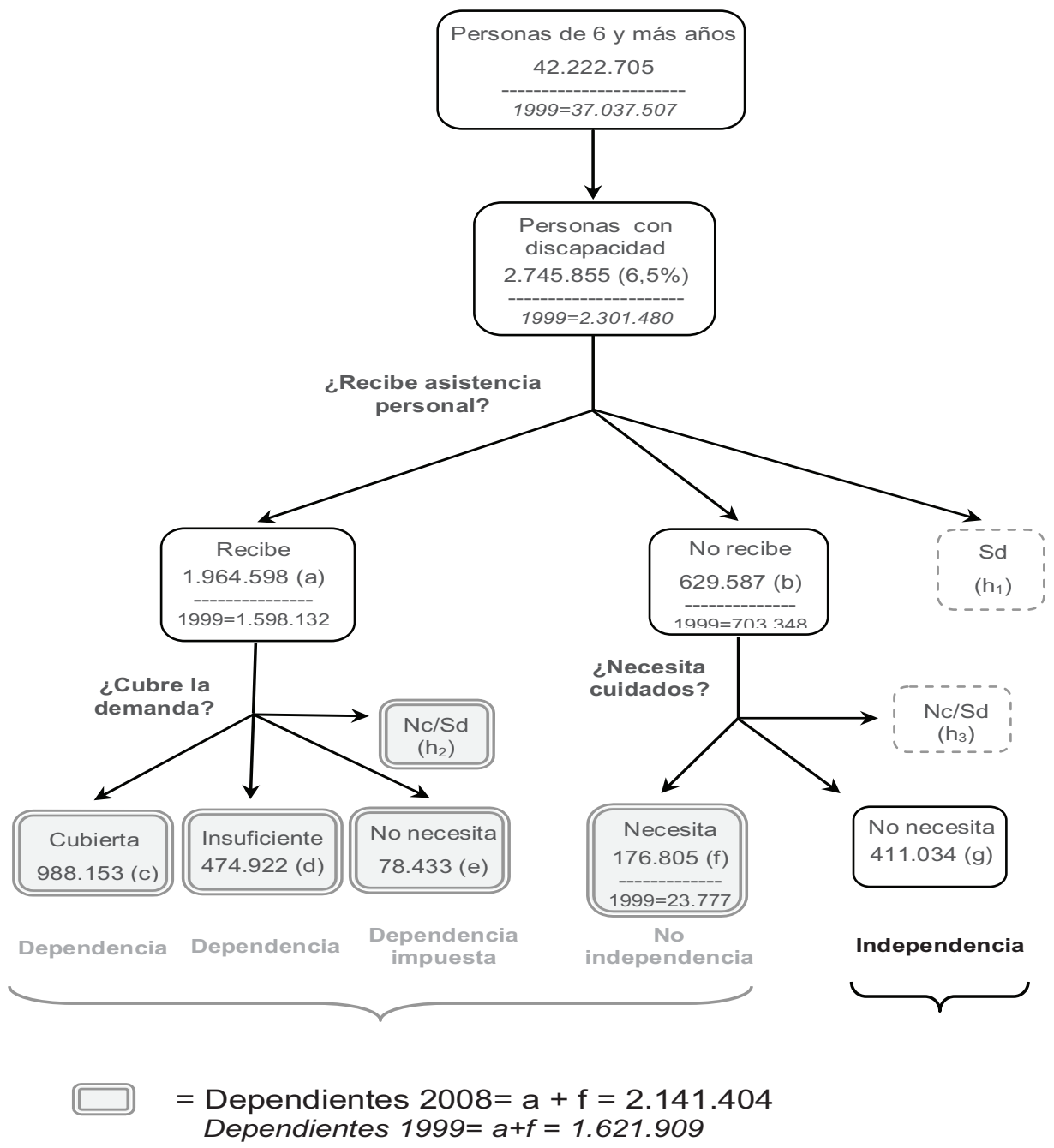

Nota: Personas en viviendas familiares. Nc/SD: no contesta y sin datos: $\mathrm{h} 1=151.670$; $\mathrm{h} 2=423.092 ; \mathrm{h} 3=41.748$.

Adicionalmente, los dependientes que residen en centros (residencias, hospitales, psiquiátricos, etc.) ascienden a 258.327 personas.

Fuente: INE: EDAD, 2008 y EDDES, 1999, microdatos. 


\section{Variaciones por sexo y edad}

Las principales características sociodemográficas de la población dependiente de 6 y más años están estrechamente relacionadas con la edad (Tabla 1). La prevalencia se incrementa casi exponencialmente a partir de los setenta años, cosa que aún se acentuaría incluyendo a los que viven en residencias colectivas, pues ocho de cada diez tienen 65 ó más años. La dependencia no alcanza al 1\% de los niños y jóvenes, es del $2 \%$ en los adultos, pero afecta al $20 \%$ de la población mayor.

Realmente existen tres perfiles sociodemográficos bastante diferenciados atendiendo a la edad y sexo. Los jóvenes con dependencia (6-19 años) son sobre todo varones; entre los adultos existe un equilibrio entre sexos, pero a partir de los 45-50 años la prevalencia femenina supera a la masculina; entre los mayores el predominio femenino, absoluto y relativo, es notable.

Tabla 1.- Características sociodemográficas de la población dependiente de 6 y más años. España, 2008

\begin{tabular}{|c|c|c|c|c|c|c|}
\hline & \multicolumn{3}{|c|}{ Edad de los dependientes } & \multirow{2}{*}{$\begin{array}{c}\text { Total } \\
\text { población } \\
\text { dependiente } \\
2008\end{array}$} & \multirow{2}{*}{$\begin{array}{c}\text { Depen- } \\
\text { dientes } \\
1999\end{array}$} & \multirow{2}{*}{$\begin{array}{c}\text { TOTAL } \\
\text { POBLACIÓN } \\
\text { ESPAÑA }\end{array}$} \\
\hline & 6-19 años & 20-64 años & $65+$ años & & & \\
\hline Dependientes 2008 & 46.742 & 632.369 & 1.462.292 & 2.141 .404 & & 42.222 .705 \\
\hline Porcentajes horizontales & 2,2 & 29,5 & 68,3 & 100 & & \\
\hline Dependientes 1999 & 55.131 & 468.793 & 1.097.986 & & 1.621 .909 & 37.037 .507 \\
\hline \multicolumn{7}{|c|}{ Porcentajes verticales sobre 2008 (en cursiva sobre 1999) } \\
\hline \multicolumn{7}{|l|}{ SEXO } \\
\hline Varón & 62,3 & 39,2 & 27,7 & 31,9 & 33,9 & 49,3 \\
\hline Mujer & 37,7 & 60,8 & 72,3 & 68,1 & 66,1 & 50,7 \\
\hline \multicolumn{7}{|l|}{ ESTADO CIVIL } \\
\hline Soltero & 100 & 32,8 & 6,9 & 16,6 & 21,2 & 39,8 \\
\hline Casado & 0,0 & 54,3 & 42,4 & 45,0 & 43,5 & 49,5 \\
\hline Viudo & 0,0 & 4,6 & 49,2 & 34,9 & 33,5 & 6,9 \\
\hline Separado/divorciado & 0,0 & 8,3 & 1,5 & 3,4 & 1,7 & 3,8 \\
\hline \multicolumn{7}{|l|}{ TIPO DE HOGAR } \\
\hline Unipersonal & 0,0 & 6,4 & 19,9 & 15,5 & & 6,8 \\
\hline Pareja & 0,0 & 17,8 & 26,3 & 23,2 & & 17,7 \\
\hline Multigeneracional & 93,6 & 65,9 & 40,5 & 49,2 & & 66,3 \\
\hline Otro tipo & 6,4 & 9,9 & 13,3 & 12,1 & & 9,2 \\
\hline \multicolumn{7}{|l|}{ ESTUDIOS } \\
\hline Analfabeto/sin estudios & 40,0 & 32,1 & 62,2 & 52,8 & 56,6 & 16,5 \\
\hline Estudios primarios & 26,5 & 29,3 & 26,3 & 27,2 & 32,6 & 25,8 \\
\hline Estudios secundarios & 6,5 & 28,7 & 7,8 & 13,9 & 8,0 & 33,9 \\
\hline Estudios superiores & 0,1 & 9,7 & 3,5 & 5,2 & 2,8 & 19,6 \\
\hline \multicolumn{7}{|l|}{ TAMAÑO MUNICIPAL } \\
\hline Menos de 10.000 hab. & 16,7 & 20,2 & 28,2 & 25,6 & 31,4 & 21,3 \\
\hline Entre 10.000 y 50.000 hab. & 30,6 & 27,0 & 22,9 & 24,3 & 24,3 & 25,9 \\
\hline Más de 50.000 hab. & 52,7 & 52,8 & 48,8 & 50,1 & 44,3 & 52,8 \\
\hline
\end{tabular}

Nota: No se muestran en la tabla las categorías NC "no contesta" y "No procede" (estudios)

FUENTE: INE. EDAD, 2008. Población de 6 y más años en viviendas familiares. EDDES, 1999. Población de 6 y más años en viviendas familiares. Elaboración propia a partir de los microdatos. 
Por ello, la dependencia tiene rasgos femeninos en conjunto (Figura 2). Dos de cada tres dependientes son mujeres $(68,1 \%)$. Puede que los hombres padezcan enfermedades con mayor grado de letalidad (debido a conductas o hábitos de mayor riesgo) y que las mujeres sufran más a menudo condiciones de salud crónicas que generan discapacidad y dependencia. También es posible que la forma de preguntar y la naturaleza de los ítems que integran la EDAD produzcan un sesgo: muchos hombres no se sienten incapacitados para realizar determinadas tareas, porque nunca las hacen. Es posible que por razones culturales el varón sea más reacio a declarar debilidad o problemas funcionales ante situaciones de naturaleza similar a las de las mujeres, o que estas tengan más trato con servicios sanitarios (por los horarios más flexibles de las tradicionales amas de casa o por los roles tradicionales en la atención a la salud de los hijos). Finalmente, la mayor longevidad de la mujer, su mayor edad media, motiva que sus tasas de dependencia sean más altas al analizar el conjunto.

Figura 2.- Pirámide de población dependiente

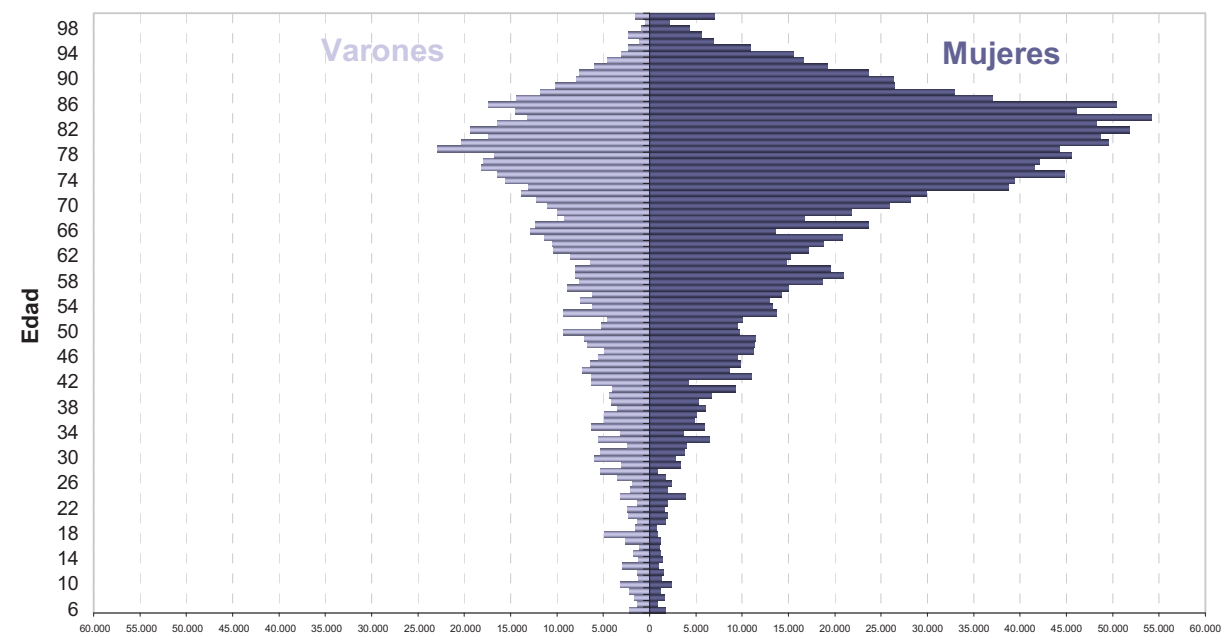

FUENTE: INE. EDAD, 2008. Población de 6 y más años en viviendas familiares.

OBSERVACIÓN: No se tienen en cuenta aquellos individuos que no han podido ser identificados como dependientes.

Entre 1999 y 2008 se dibuja una mayor dependencia entre las mujeres (representaban entonces el $66,1 \%$ de todos los dependientes), y especialmente en el conjunto del grupo de edad de 65 y más años, que también ve crecer la proporción de dependientes entre ambas fechas. A falta de estudios de detalle, esta diferencia puede ser debida a los cambios metodológicos entre ambas encuestas, pero también a la diferente consideración de "ayuda" recibida, o incluso a cambios en la estructura por edad. 


\section{Otras características sociodemográficas}

Tipo de hogar.- La frecuencia del apoyo de los hijos a los dependientes de más edad guarda estrecha relación con la corresidencia; solteros, viudos o separados tienen más probabilidad a vivir en hogares unipersonales y tienen más difícil el apoyo familiar si precisan cuidados.

Puesto que el estado civil y las formas de convivencia están relacionados con la edad, todos los jóvenes dependientes que encontramos están solteros, los adultos son casados en su mayoría y en la vejez la mayor parte son viudos. La proporción de personas dependientes que no conviven con sus descendientes crece rápidamente con la edad. Es igualmente destacable que el 45,3\% de los dependientes de 65 y más años viva en hogares en los que ellos mismos son la persona de referencia. Además, cuanto mayor es la edad del dependiente menor es el tamaño medio del hogar.

Muy probablemente la cada vez mayor proporción de quienes sobreviven hasta los 65 años, y el constante descenso de la mortalidad también entre los más mayores, está siendo un factor acrecentador de la presencia de discapacidades y dependencia. Sin duda también así se explica que entre 1999 y 2008 se observe un ligero aumento de la proporción de viudez (mayoritariamente femenina) entre los dependientes, pese a que la edad media a la viudez se esté retrasando también con las mejoras en la mortalidad.

Dependencia y soledad.- A pesar de la creciente heterogeneidad de las formas familiares de convivencia, se apunta una tendencia hacia una mayor soledad residencial de los mayores y en concreto de los dependientes de edad; casi trescientos mil de éstos viven ahora en hogares unipersonales (80.000 más que en 1999), y suponen ya un $20 \%$ de todos los dependientes mayores.

Ingresos.- Los hogares unipersonales, con persona dependiente, son los que se encuentran entre los de menos ingresos; $87,1 \%$ de los unipersonales obtienen menos de mil euros mensuales. Esta tendencia se acentúa con la edad, por lo que los hogares unipersonales de personas dependientes mayores son los que tienen los menores ingresos. Los hogares multigeneracionales, con dependiente, se distribuyen entre los diferentes niveles de ingresos.

Nivel de estudios.- La dependencia correlaciona inversamente con el nivel de instrucción. La proporción de dependientes entre quienes no saben leer ni escribir (sobre todo población de 65 y más años) o no tienen estudios es casi diez veces superior que la existente entre aquellos que tienen estudios superiores (no reflejado en la tabla). Esta tendencia aún es más fuerte entre la población de edad, donde las diferencias de instrucción son más abultadas. Por supuesto, los estudios tienen un efecto directo en las capacidades propias y la posibilidad de aprovechar recursos para afrontar problemas de salud, pero también son un indicador indirecto de muchos otros factores como la posición económica y social, y los estándares y condiciones de vida asociados. Este es un factor de optimismo respecto al futuro, porque el simple relevo generacional juega a favor de una rápida elevación del nivel de estudios entre los más mayores.

Distribución territorial.- La distribución geográfica de la dependencia en España mantiene de forma gráfica dos grandes áreas que repiten el modelo del mapa de 1999. Una diagonal noroeste-sudeste deja al sur una España con prevalencias superiores a la 
media, destacando Galicia, Murcia y Extremadura, con las más altas; a ellas se añade Andalucía, Asturias, Castilla-León y Castilla-La Mancha. En la otra parte de la diagonal, con prevalencias más bajas, se sitúan Madrid, Cataluña, País Vasco, Navarra, La Rioja, las Islas Baleares, además de Canarias.

La evolución general de la distribución de la población en el territorio, cada vez más urbana, se traduce en que ahora la mitad de los dependientes de todas las edades vivan en las capitales de provincia y municipios de más de 50.000 habitantes, y hasta tres de cada cuatro dependientes vivan en el conjunto de municipios urbanos. Por su parte, el mundo rural (municipios de menos de 10.000 habitantes) envejece a fuerte ritmo y sus proporciones de población dependiente aumentan ligeramente entre 1999 y 2008 (de 5,5\% a 6,1\% de toda la población que vive en ese umbral de hábitat); medio millón de personas que necesitan ayuda personal viven en municipios rurales. Por tanto el reto es doble: elevada prevalencia en un mundo rural con poca población y recursos, y elevado volumen en entornos urbanos (1,6 millones incluyendo los municipios urbanos pequeños de 10-50.000 habitantes).

\section{Prevalencia de las limitaciones en las actividades de la vida diaria}

Los tipos de incapacidad funcional más extendidos como causa de dependencia están determinados por el peso que tienen las personas mayores en el cómputo general (Figura 3).

Los problemas funcionales para movilidad externa son los más frecuentes; impiden a las personas manejarse en la comunidad, perdiendo las ventajas de vivir en sociedad y desarrollar su vida y actividades de relación en una parte del espacio físico próximo. 
Figura 3.- Tipo de discapacidad por edad

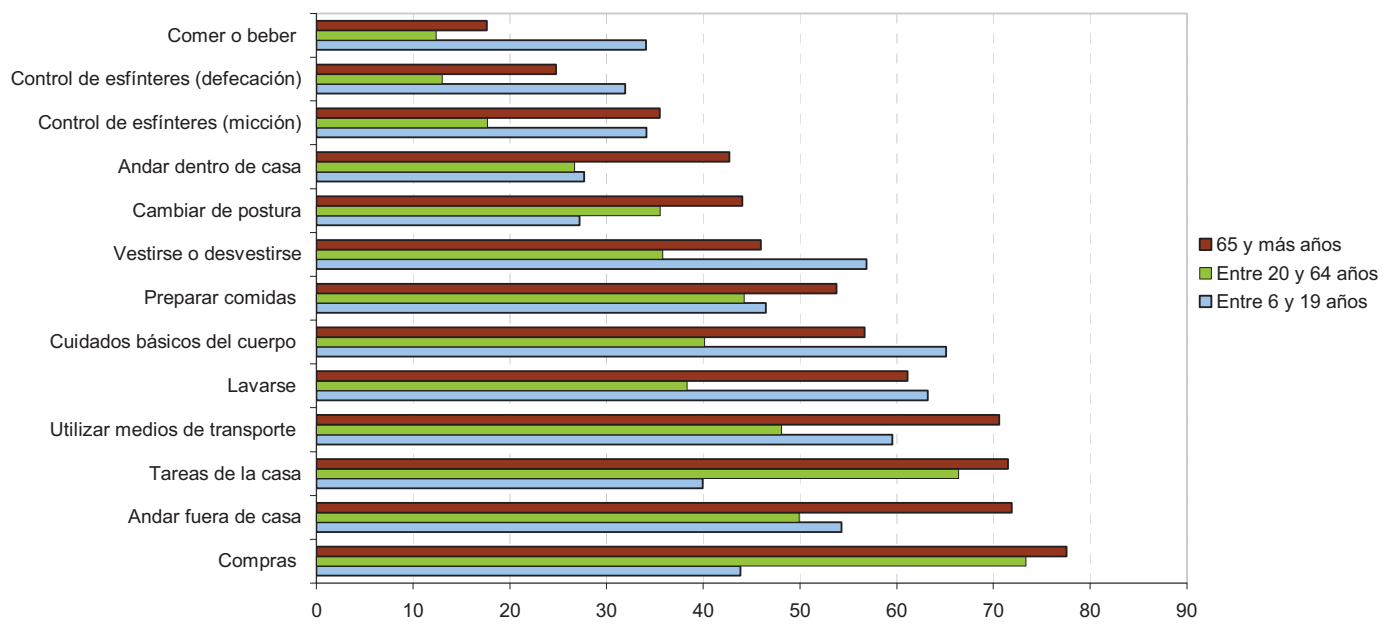

FUENTE: INE. EDAD, 2008. Población de 6 y más años en viviendas familiares.

NOTA: Porcentaje de dependientes que declara dificultad en cada una de las actividades.

Más de un millón de dependientes declara problemas funcionales relacionados con las actividades de movilidad exterior, que son generalmente las primeras habilidades que se pierden cuando se inicia el proceso de discapacidad: realizar compras (lo que implica movilidad y cierta habilidad cognitiva), andar fuera de la vivienda, en el entorno de la comunidad y utilizar medios de transporte.

Detrás se sitúan otras actividades instrumentales, como realizar las tareas domésticas, que son necesarias para mantener cierta autonomía en el hogar. En éstas existe un sesgo por sexo; las mujeres declaran problemas en doble proporción que los varones para tareas domésticas $(4,9 \%$ y $2,0 \%$ sobre el conjunto de la población, respectivamente); muchos varones no declaran problemas pues no son tareas que habitualmente realicen.

El siguiente conjunto de dificultad en actividades, que afecta a menos personas pero con mayor gravedad, se relacionan con la independencia personal; son las actividades básicas o de autocuidado (lavarse, cuidados básicos del cuerpo, vestirsedesvestirse). Entre 600-800.000 dependientes señalan estos problemas.

Finalmente, las incapacidades que afectan a menor número de personas, 250-300.000, pero son las de naturaleza más grave y que significan mayor carga de atención, son las relacionadas con el control de esfínteres y la dificultad importante para llevar a cabo tareas de comer o beber sin ayuda y sin supervisión, también incluidas en las actividades básicas.

Entrada en discapacidad.- El orden de adquisición de las discapacidades parece coincidir con el escalamiento jerárquico de las funciones sociobiológicas, de forma que las personas entran primero en discapacidad en las funciones más complejas o de 
rango superior, como las instrumentales, y acaban viendo afectadas las más básicas y sencillas (Katz y Akpom, 1976; Dunlop, Hughes y Manheim, 1997; Barberger-Gateau, Rainville, Letenneur y Dartigues, 2000; Verbrugge, Yang y Juarez, 2004). Esta pauta explica que haya más personas afectadas en las funciones complejas y menos en las básicas, y arroja información valiosa para la organización de planes de ayudas.

A falta de estudios de escalamiento y de carga de discapacidad más precisos, este supuesto parece confirmado en nuestros datos. Comprar, realizar tareas de casa y movilidad exterior (andar, tomar el autobús) están entre primeras actividades afectadas: el $63,8 \%$ de los dependientes declara que comprar fue la primera en las que tuvo problemas y precisó ayuda, y son más de la mitad de los dependientes los que señalan dificultades en esas actividades (Tabla 2). En consonancia con ese principio, los problemas para comer sólo se señalan como primera discapacidad adquirida por el $13,4 \%$ de los dependientes, seguido por el control de esfínteres $(17,0 \%$ defecar; $22,1 \%$ orinar). Considerando sólo a los mayores, el patrón es aún más claro: las dificultades empiezan por la realización de la compra $(63,4 \%)$, andar fuera en la comunidad $(59,2 \%)$, tomar el autobús $(58,8 \%)$ y realizar compras $(58,4 \%)$; en el otro extremo se repite el orden de la aparición de problemas citado.

Tabla 2.- Dificultad adquirida en primer lugar

\begin{tabular}{|l|c|c|c|c|}
\multicolumn{1}{c|}{$\begin{array}{c}\text { Actividades de la vida diaria (unidades: } \\
\text { porcentajes) }\end{array}$} & $\begin{array}{c}\text { Entre } \mathbf{6} \text { y } \mathbf{~ 1 9} \\
\text { años }\end{array}$ & $\begin{array}{c}\text { Entre 20 y } \mathbf{6 4} \\
\text { años }\end{array}$ & $\begin{array}{c}\mathbf{6 5} \text { y más } \\
\text { años }\end{array}$ & TOTAL \\
\hline Compras & 41,5 & 66,2 & 63,4 & 63,8 \\
Andar fuera de casa & 53,2 & 45,0 & 59,2 & 54,9 \\
Utilizar medios de transporte & 57,8 & 43,6 & 58,8 & 54,3 \\
Tareas de la casa & 38,1 & 59,9 & 58,4 & 58,4 \\
Lavarse & 60,0 & 34,0 & 48,3 & 44,3 \\
Cuidados básicos del cuerpo & 62,9 & 35,6 & 44,6 & 42,3 \\
Preparar comidas & 44,5 & 40,1 & 43,9 & 42,8 \\
Cambiar de postura & 27,3 & 32,3 & 36,3 & 34,9 \\
Vestirse o desvestirse & 53,3 & 31,5 & 35,6 & 34,8 \\
Andar dentro de casa & 27,7 & 23,8 & 35,1 & 31,6 \\
Control de esfínteres (micción) & 33,9 & 15,1 & 24,8 & 22,1 \\
Control de esfínteres (defecación) & 30,9 & 11,9 & 18,8 & 17,0 \\
Comer o beber & 33,7 & 11,2 & 13,8 & 13,4 \\
\hline
\end{tabular}

NOTA: Porcentaje de personas que declara haber adquirido esa actividad en primer lugar.

FUENTE: INE. EDAD, 2008. Población de 6 y más años en viviendas familiares.

Entre los jóvenes la forma de entrada presenta un modelo diferente. Muchos de los problemas funcionales se adquieren de forma simultánea, como suelen producir los accidentes o los problemas congénitos. Es en adultos y mayores donde se observa el escalamiento propio de los procesos de discapacidad progresiva (Ferrucci et al, 1996). Un indicador confirmatorio es el tiempo medio transcurrido desde la entrada en la primera discapacidad hasta la última. Entre los jóvenes, el tiempo medio es mínimo (0.4 años), mientras que en los mayores es de 3.7 años. 
El mismo patrón de ordenación o escala se observaba ya en 1999, con la salvedad de que en las actividades de movilidad exterior estaba más acentuada la dificultad para desplazarse utilizando medios de transporte y andar fuera. Pero ambas encuestas coinciden en que los dependientes mayores se ven más afectados en todas las actividades a excepción de las más básicas (comer, control de esfínteres, vestirse), en las que son los jóvenes afectados los que más dificultad tienen para su realización, de forma consonante con la diferente forma de entrar en discapacidad.

\section{Principales enfermedades asociadas a la dependencia}

Muchas de las discapacidades que originan dependencia tienen su origen en ciertos problemas de salud. Por tanto, los cambios en la prevalencia de tales problemas son importantes para explicar las variaciones en la prevalencia de ciertos tipos de discapacidad y dependencia (Tabla 3).

Como acaba de señalarse, entre los más jóvenes los problemas de salud que generan dependencia suelen ser congénitos; el $19,4 \%$ de los dependientes tienen daño cerebral adquirido, parálisis cerebral $(14,4 \%)$ o síndrome de Down $(8,1 \%)$, o enfermedades raras, autismo o distrofia muscular.

Tabla 3.- Principales enfermedades de los dependientes, por edad.

\begin{tabular}{|l|l|l|}
\hline 6-19 años & 20-64 años & 65 y más años \\
\hline Daño cerebral $(19,4 \%)$ & Dolor de espalda $(48,7 \%)^{*}$ & Artritis/artrosis $(65,8 \%)$ \\
Enfermedades raras $(19,0 \%)$ & Hepresión $(41,1 \%)$ & Hipertensión $(43,3 \%)^{*}$ \\
Parálisis cerebral $(14,4 \%)$ & Cataratas $(37,5 \%)$ \\
Autismo $(12,5 \%)$ & Hipertensión $(24,3 \%)^{*}$ & Depresión $(26,7 \%)$ \\
Distrofia muscular $(12,2 \%)$ & Colesterol $(22,2 \%)^{*}$ & Colesterol $(25,8 \%)^{*}$ \\
Síndrome de Down $(8,1 \%)$ & Artritis reumatoide $(19,5 \%)$ & Artritis reumatoide $(25,7 \%)$ \\
& Distrofia muscular $(14,8 \%)$ & Diabetes $(23,0 \%)^{*}$ \\
& & Infarto de miocardio $(18,0 \%)$ \\
& & Accidentes cerebrovasculares $(14,5 \%)$ \\
\hline
\end{tabular}

Nota: Porcentaje de dependientes que declaran enfermedades diagnosticadas (módulo B); * problemas crónicos declarados (módulo K).

Fuente: INE: EDAD, 2008. Población de 6 y más años en viviendas familiares.

Entre los adultos ya empiezan a aparecer enfermedades crónicas, degenerativas u otras condiciones de salud provocadas por malos hábitos o circunstancias laborales. El $48,7 \%$ de los dependientes declara dolor de espalda y al $38,8 \%$ se le ha diagnosticado artritis/artrosis o sufre de hipertensión o colesterol elevado.

Entre los mayores, los problemas osteomusculares, resultado de procesos degenerativos, son los más importantes: $65,8 \%$ de los dependientes tienen diagnosticada artritis/artrosis, un $25,7 \%$ artritis reumatoide, enfermedad no letal pero con muy alto efecto discapacitante. Los problemas de corazón y de circulación son otra segunda gran causa: el $18,0 \%$ han sido diagnosticados de infarto de miocardio y $14,5 \%$ de accidentes cerebrovasculares; son enfermedades de alta letalidad y, si se superan, suelen tener secuelas discapacitantes, además de presentarse junto a otras condiciones 
adversas como hipertensión y colesterol. La diabetes también está estrechamente asociada a discapacidad física y dependencia, pues afecta a movilidad y otras funciones; algo similar sucede con las cataratas, que limitan el desempeño de actividades cotidianas. Con importancia creciente, comparado con otras estadísticas, aparecen enfermedades mentales degenerativas como el Alzheimer $(10,6 \%) \mathrm{u}$ otras demencias $(8,0 \%)$; lógicamente, la prevalencia de enfermedades cognitivas severas en personas dependientes es mucho mayor en las que residen en instituciones $(15,1 \%$, y $23,1 \%$, respectivamente), pero con el aumento de la edad se incrementan notablemente estos problemas degenerativos tanto en personas que viven en instituciones como en viviendas familiares.

\section{Severidad}

De acuerdo con nuestra delimitación de discapacidad y la medida de gravedad adoptada, un $15,3 \%$ de los dependientes tienen un severidad casi inapreciable (hasta 0.04 en una escala de $0-1)$ y un $32,3 \%$ es leve $(0.05-0.24 \%)$ (Tabla 4$)$. El resto presenta una severidad que hemos estimado entre moderada y grave o total, aproximadamente medio millón de personas en cada una de estas dos categorías.

Las situaciones más severas se encuentran entre los mayores: 463.102 dependientes con severidad grave o total, de un total de 577.577 dependientes graves. También entre los jóvenes destacan las situaciones de máxima severidad, aunque su cifra absoluta es baja.

Tabla 4.- Gravedad por edad de las personas de 6 y más años dependientes

\begin{tabular}{|l|c|c|c|c|}
\cline { 2 - 4 } \multicolumn{1}{c|}{} & \multicolumn{3}{c|}{ Edad de los dependientes } & \multirow{2}{*}{ TOTAL } \\
\cline { 2 - 4 } \multicolumn{1}{c|}{} & $\mathbf{6 - 1 9}$ años & $\mathbf{2 0 - 6 4}$ años & $\mathbf{6 5 +}$ años & \\
\hline Insignificante & 14,3 & 21,2 & 12,8 & 15,3 \\
Leve & 32,7 & 40,7 & 28,7 & 32,3 \\
Moderada & 19,8 & 20,5 & 25,6 & 24,0 \\
Grave o total & 29,1 & 16,0 & 31,7 & 27,0 \\
Sin datos & 4,1 & 1,7 & 1,2 & 1,4 \\
\hline Dependientes (N) & $\mathbf{4 6 . 7 4 2}$ & $\mathbf{6 3 2 . 3 6 9}$ & $\mathbf{1 . 4 6 2 . 2 9 2}$ & $\mathbf{2 . 1 4 1 . 4 0 4}$ \\
\hline
\end{tabular}

Nota: Porcentajes verticales.

FUENTE: INE. EDAD, 2008. Población de 6 y más años en viviendas familiares.

La severidad de la dependencia y otras circunstancias personales (edad, estado civil, otras condiciones de salud) y familiares condicionan la forma de convivencia (Figura 4). Los dependientes más graves no suelen vivir solos, aunque lo hace el 8,7\% y son sobre todo personas de edad. Ese mismo patrón de severidad se repite en los casos moderados.

En los hogares multigeneracionales es donde reside el mayor número de dependientes y además donde se presentan las situaciones de mayor severidad; en los que viven personas de edad se dan ambas circunstancias acentuadas. Entre los dependientes jóvenes es el tipo de hogar más extendido (en cualquier categoría de severidad). 
Entre los mayores, ese tipo de hogar significa la presencia de algún hijo/a en tareas de atención como se verá más adelante.

Entre 1999 y 2008 se mantiene la tendencia de situaciones más graves en personas mayores y jóvenes; en conjunto las situaciones de máxima gravedad (no puede realizar la actividad) se han acentuado a la vez que descienden las situaciones de severidad moderada y severa ${ }^{7}$.

Figura 4.-Tipo de hogar en el que viven los dependientes según su gravedad

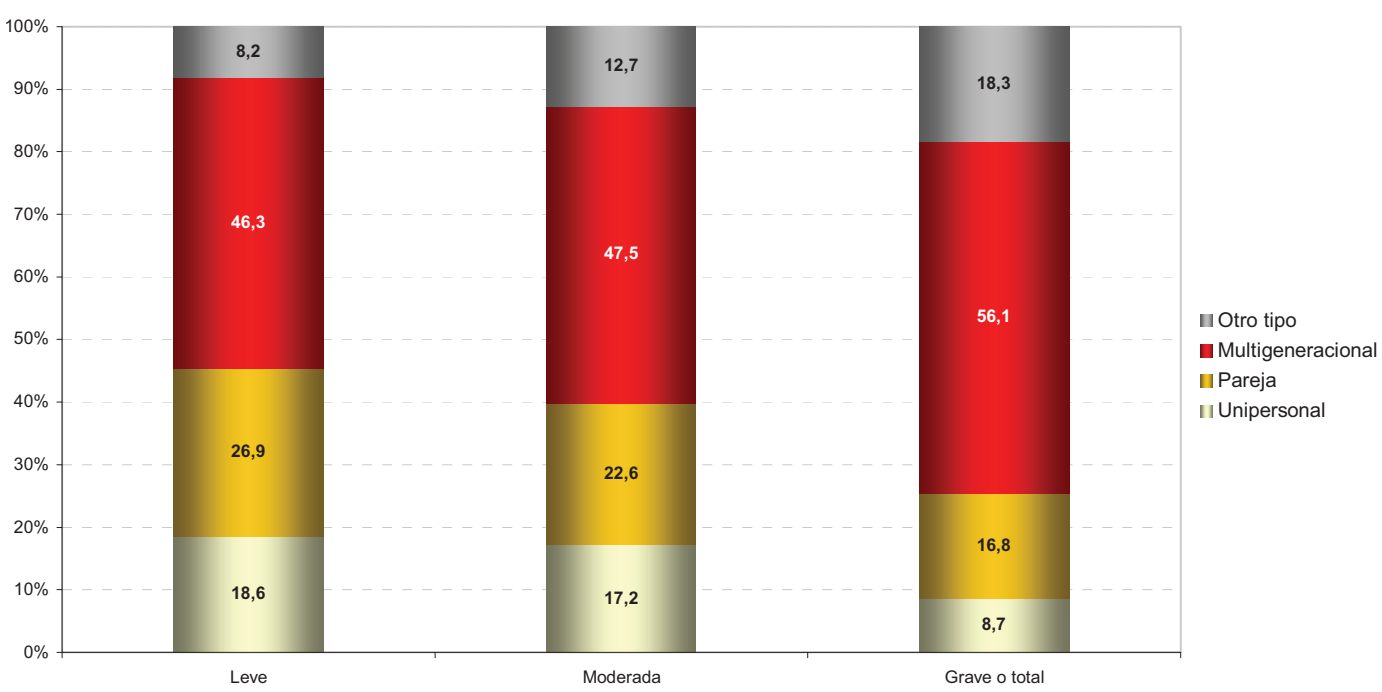

FUENTE: INE. EDAD, 2008. Población de 6 y más años en viviendas familiares.

Se agrupan las categorias insignificante y leve $(0-0,24$ de la escala de $0-1)$.

${ }^{7}$ Para esta comparación de gravedad entre las dos encuestas, EDDES y EDAD, se ha utilizado la misma clasificación de severidad codificada por el INE (y diferente de nuestra propia estimación de severidad, utilizada hasta aquí) y para las mismas actividades seleccionadas (13); según esta clasificación para 2008 , un $61,1 \%$ de los dependientes declaran una severidad total, un $24,3 \%$ dificultad severa, $12,8 \%$ moderada; en 1999 , los porcentajes eran $49,6 \%, 31,7 \%$ y $17,8 \%$, respectivamente. La razón podría residir, como ya se ha indicado antes, en el aumento del número y proporción de personas de mucha edad, con mayor dependencia y gravedad declaradas. Pero la comparación debe ser tomada con precaución pues responde a otra metodología y también puede haberse elevado el estándar social de salud y la solicitud de ayudas para situaciones similares de discapacidad funcional. 


\section{La recepción de ayuda}

El otro polo que define la dependencia, junto a los problemas de incapacidad funcional, es la recepción o demanda de ayuda personal. Los cuidados personales se han computado sobre las personas con discapacidad y los reciben la inmensa mayoría de los dependientes, aunque una mínima proporción no recibe asistencia pese a solicitarla. Como se verá, el patrón sociodemografico de las ayudas es muy estable en el tiempo.

El curso de vida y el cuidado: madre, esposa e hija.- Existe un patrón de cuidados puesto de manifiesto en diferentes estudios basados en encuestas, especialmente las hechas a personas mayores (Puga, Abellán y Sancho, 2006; Imserso, 2006; I.E.A., 2006; Abellán, Castejón, Esparza, 2007). La ayuda informal es la base de la atención a los dependientes, sin apenas cambios desde la anterior encuesta de 1999. Se repite el modelo tradicional con la mujer como responsable principal de la tarea de cuidados; es la cuidadora principal en siete de cada diez casos en que puede establecerse el género del cuidador ( 956.232 cuidadoras principales sobre 1.307 .433 personas cuidadoras); habría que sumar además empleados del hogar y otros que trabajan para los servicios sociales, así como otros familiares (normalmente mujeres) y otros tipos, y con estos grupos se alcanza la cifra de 1.960 .724 personas cuidadas (no necesariamente coinciden con la cifra de cuidadores, pues puede haber un cuidador que atienda a dos personas dependientes); el resto hasta el número total de dependientes son aquellos que demandan pero no reciben asistencia.

La distinta situación conyugal y de convivencia de varones y mujeres dependientes explica en parte este patrón de cuidado. Generalmente, el varón tiene mayor edad que su pareja y, por tanto, mayor probabilidad de necesitar ayuda para una limitación funcional. La diferencia de edad, sumada a la mayor esperanza de vida femenina, hace más frecuente que sea la mujer la que sobrevive al marido. En caso de dependencia se recurre a la red familiar, principalmente la hija, que asume la tarea en desigual reparto con sus hermanos varones, por lo que se repite el esquema de género en el cuidado.

Un repaso de la forma de recepción de ayuda en las diferentes etapas del curso de vida permite delinear un modelo de familia española cuidadora con fuertes lazos, como sucede en otros países del sur de Europa, en contraste con países del centro y norte europeo.

Pero la fortaleza de los vínculos familiares esconde una realidad; se asienta principalmente en la mujer. En las tres etapas del curso de vida en que se ha clasificado a los dependientes predomina como cuidadora, bien como madre de un hijo dependiente (6-19 años), como madre, esposa o hija de un varón con discapacidad funcional (20-64 años) o como hija de padres (sobre todo, madre) dependientes de edad avanzada (Figura 5). Este perfil de hija es el más representativo del cuidado, con la cifra más alta (463.356 hijas cuidando a dependientes, sobre todo mayores), dos veces superior a la de esposas y tres veces más alta que la de madres cuidadoras. 
Figura 5.- Quién cuida a los dependientes en función de la edad de los mismos

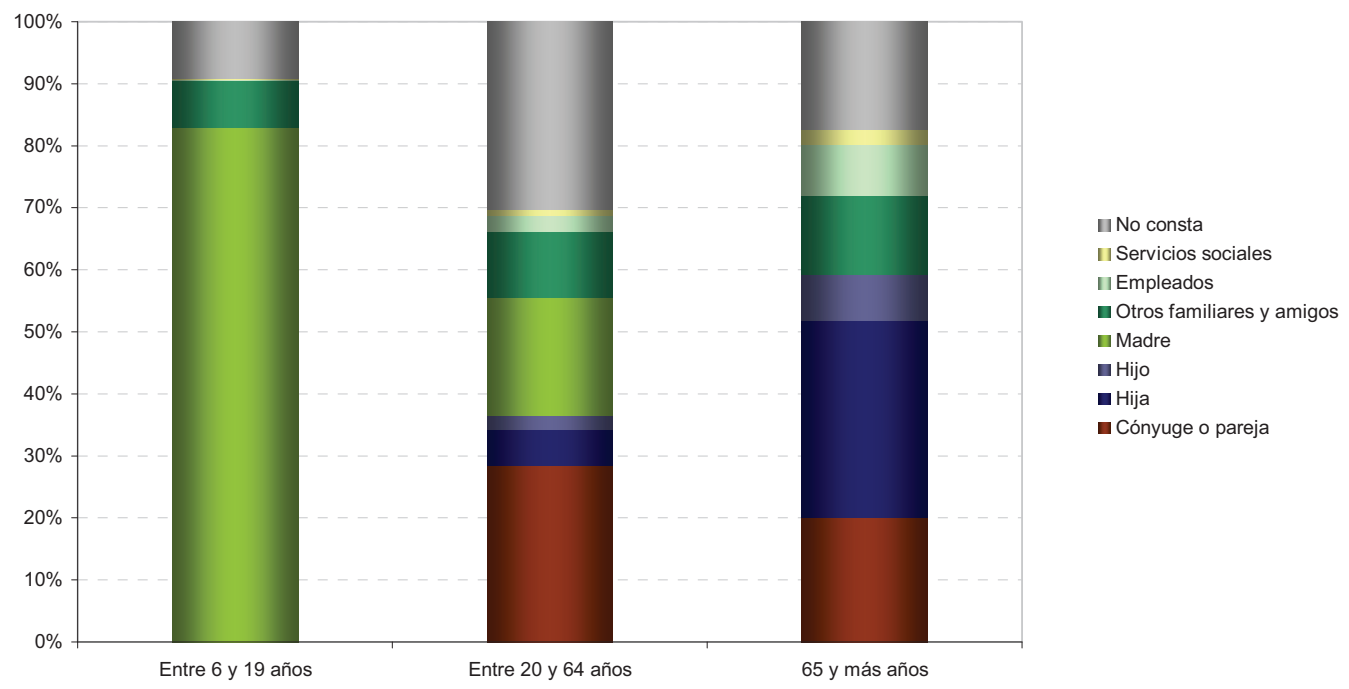

FUENTE: INE. EDAD, 2008. Población de 6 y más años en viviendas familiares.

Las cuidadoras son mujeres de edad intermedia; 42 años para las cuidadoras de jóvenes, 53 para las cuidadoras de adultos y 52 para las hijas cuidadoras de mayores. Es decir, predomina la mujer que ya supera los 50 años, con la menopausa en ciernes, y aún no finalizadas totalmente las tareas reproductivas (hijos aún no emancipados). A menudo esta situación provoca tensiones para mantener la trayectoria laboral hasta la edad normal de jubilación.

Los dependientes mayores son los más numerosos. Los hombres son cuidados ante todo por su mujer, seguida por una hija, corresidente o no, y en menor proporción por un hijo. Los servicios sociales tienen un escaso papel tanto en este escenario como cuando es la mujer la persona dependiente. La mujer dependiente es cuidada principalmente por su hija, y a continuación por su marido, si está casada; el hijo y la empleada, doméstica interna o que presta servicio desde fuera del hogar, vienen a continuación ${ }^{8}$.

El modelo de cuidado de los dependientes de más edad según el tipo de hogar es un buen ejemplo de cómo se relacionan convivencia, ayuda informal, carga del cuidado y aparición de otras formas de prestación de ayuda formal (empleados y servicios sociales) (Figura 6).

${ }^{8}$ En el término genérico "personas empleadas" se incluyen empleadas de hogar residentes, otras personas empleadas de hogar y empleadas profesionales sociosanitarias $(10 \%$ aproximadamente del rubro). 
Figura 6.- Quién cuida a los dependientes de 65 y más años según el tipo de hogar en el que viven

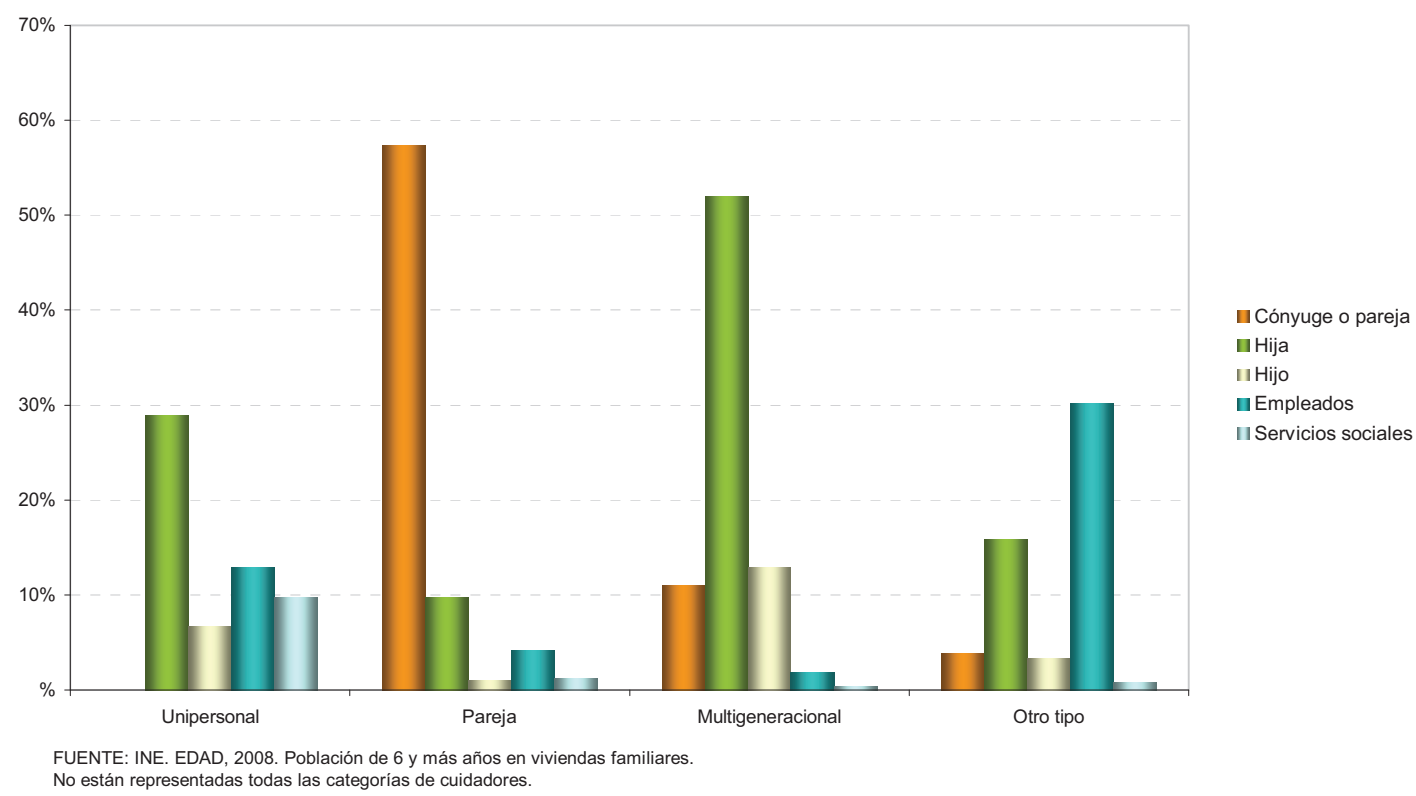

En los hogares unipersonales cuidan principalmente una hija, una empleada en funciones de servicio doméstico y cuidadora o un profesional sociosanitario, y tienen presencia los servicios sociales. En los hogares donde vive una pareja, los dependientes son cuidados por el cónyuge y en menor proporción por una hija (no corresidente). En los hogares multigeneracionales, la cuidadora es una hija en proporción cuatro veces superior a un hijo; los servicios sociales apenas tienen relevancia. En los hogares constituidos de otra forma, sin vínculo filial ni conyugal, son las personas empleadas y la hija (residiendo fuera) las que llevan el peso de la tarea.

Los servicios sociales, escasos aún en el cuidado, podrían estar discriminando los hogares según la forma de convivencia, y habrá que ver si la Ley de Dependencia (Ley, 2006) va a modificar esta pauta; su intervención se centra en aquellos hogares menos "familiarizados", con personas de especial vulnerabilidad conferida por su soledad residencial, dejando a cargo de los familiares el resto de situaciones de dependencia, a veces más invisibles precisamente por ello.

Empieza a revelarse en EDAD la importancia creciente del cuidador principal extranjero (sobre todo mujer). En 1999, no llegaba al 1\% los extranjeros que trabajaban 
como empleados de hogar y cuidaban. Ahora casi alcanza el 6\%, algo más de cien mil personas 9

\section{Conclusiones}

La definición de dependencia usada en este trabajo adopta el enfoque de la incapacidad funcional y la naturaleza social de la dependencia. Implica ambas dimensiones: dificultad en las actividades de la vida diaria y una relación social de recepción de ayuda por parte de otra persona.

Dicha definición se ha hecho operativa sobre los datos primarios de la Encuesta sobre Discapacidad, autonomía personal y situaciones de Dependencia (EDAD), llevada a cabo por el INE en 2008. Dicha aplicación arroja un número de 2.141.404 personas dependientes de 6 y más años, que suponen el 5,1\% de la población. Ambos datos son superiores a los de 1999, aunque las diferencias metodológicas entre ambas encuestas impiden distinguir la parte producida por el proceso de envejecimiento global y el de los ya mayores.

La distribución geográfica de la dependencia refleja el sostenido proceso de urbanización poblacional, pero también el abandono juvenil de las áreas rurales (más problemático si redunda en la pérdida de apoyos familiares para los más mayores).

Los dependientes, especialmente los más graves, suelen vivir en hogares multigeneracionales y en otros sin estructura filial ni conyugal. La soledad residencial avanza progresivamente entre las personas de edad.

Se apunta que, mayoritariamente, el orden temporal de entrada en discapacidades y dependencia es jerárquico. Son las capacidades más complejas pero menos esenciales las primeras y las más frecuentemente afectadas, y la gradación continúa hasta llegar a las discapacidades menos frecuentes pero que afectan las funciones más básicas de autocuidado.

Los problemas osteomusculares (artritis/artrosis y otros) son los más frecuentes entre los dependientes; no son letales pero dificultan la movilidad y la realización de tareas de casa e incluso actividades básicas de cuidado personal. Las enfermedades circulatorias suelen tener efectos severos sobre la discapacidad cuando no son letales. Finalmente, las enfermedades mentales degenerativas (Alzheimer y otras demencias) forman otro núcleo de enfermedades diagnosticadas en aumento progresivo.

En definitiva estamos ante un ámbito, el de la convivencia y los cuidados entre personas, donde se expresan más claramente las transferencias sociales de recursos. La escasa distancia entre las dos encuestas utilizadas y, sobre todo, su difícil comparabilidad, no son óbice para constatar que la respuesta a la demanda de cuidados no

${ }^{9}$ Existe un subregistro de este fenómeno; entre las personas dependientes que reciben asistencia personal, en al menos uno de cada cinco casos no consta la nacionalidad del cuidador principal. 
ha abandonado el patrón tradicional, basado en la ayuda informal a cargo de la familia, con un papel principal de la mujer. Los empleados de hogar sólo destacan en los hogares sin estructura filial o conyugal, y es creciente la participación de la inmigración extranjera en este sector de empleo. Los servicios sociales siguen sin jugar un papel relevante.

Estas estructuras de atención y relación pueden cambiar en el futuro tal y como lo está haciendo las formas familiares. Las cohortes españolas adultas y jóvenes siguen una tendencia más parecida a la europea en comportamientos reproductivos (caída de la fecundidad y menos hijos por mujer), en nivel educativo (mujeres integradas en el sistema e incluso superando a los varones en formación), e integración en el mercado laboral (crecientes tasas de ocupación femenina). También se atisba un cambio en los roles masculinos, especialmente después de la jubilación. Pero la persistente limitación de los servicios formales a un papel prácticamente residual puede acabar por constreñir la modernización de los roles de género, la plena igualdad femenina e, incluso, la viabilidad de la familia como agencia de cuidados. No ha transcurrido el tiempo suficiente desde la aprobación de la Ley de Dependencia como para observar sus efectos, y saber si esta es una situación que en el futuro cambiará sustancialmente.

\section{Bibliografía}

Abellán García, A.; Esparza Catalán, C. (2007): “¿Qué es la dependencia? Geografía y prospectiva de la dependencia en España". Análisis Local, vol. 70, 1, p. 7-19.

Abellán García, A.; Castejón Villarejo, P.; Esparza Catalán, C. (2007): "La dependencia en las personas mayores". Sociedad y Utopía, 30, p. 133-145.

Albarrán Lozano, J.; Alonso González, P. (2006): “Clasificación de las personas dependientes a partir de la Encuesta de Discapacidades, deficiencias y estado de Salud de 1999. Revista Española de Salud Pública, 80, p. 349-360.

Alegre Escolano, A. et al. (2005): "Tasa de dependencia de la población española no institucionalizada y criterios de valoración de la severidad". Revista Española de Salud Pública, 79, p. 351-363.

Barberger-Gateau, P.; Rainville, C.; Letenneur, L.; Dartigues, J.F. (2000): “A hierarchical model of domains of disablement in the elderly: a longitudinal approach". Disability and Rehabilitation, vol. 22, 7, p. 308-317.

Béland, F.; Zunzunegui, M.V. (1999): "Predictors of functional status in older people living at home". Age and Ageing, 28, p. 153-159.

Börsch-Supan, A. (coord.) (2005): Health, Ageing and Retirement in Europe (SHARE). Mannheim, $370 \mathrm{p}$. 
Cabrero García, J. (2007): "Indicadores de discapacidad en la Encuesta de discapacidades, deficiencias y estado de salud”. Revista Española de Salud Pública, 81, p. 167-181.

Dunlop, D.D., et al. (1997): "Disability in Activities of Daily Living: Patterns of Change and Hierarchy of Disability". Am. J. Public Health, vol. 87, 3, p. 378-383.

Ferrucci, et al. (1996): "Progressive versus Catastrophic Disability: a Longitudinal View of the Disablement Process". The Journals of Gerontology, 51A, 3, M123M130.

Gignac, M.A.M.; Cott, Ch. (1998): "A conceptual model of independence and dependence for adults with chronic physical illness and disability". Social Science and Medicine, vol. 47, 6, p. 739-753.

Gispert Magarolas, R. et al (2008): "El perfil de la dependencia en España: un análisis de la encuesta de Discapacidades de 1999". Revista Española de Salud Pública, 82, p. 653-665.

IEA -Instituto de Estadística de Andalucía- (2006), Andalucía. Dependencia y solidaridad en las redes familiares. Sevilla: IEA. Consejería de Economía y Hacienda de la Junta de Andalucía.

IMSERSO (1994): Clasificación Internacional de Deficiencias, Discapacidades y Minusvalias (CIDDM, 1980). Madrid, Imserso, 279 p.

IMSERSO (2004): Libro Blanco. Atención a las personas en situación de dependencia en España. Madrid, Imserso, 820 p.

IMSERSO (2006): A propósito de las condiciones de vida de las personas mayores. Encuesta 2006. Madrid, Imserso, 166 p. Disponible en:

http://www.imsersomayores.csic.es/documentos/documentos/imserso-proposito01.pdf

INE: Encuesta sobre Discapacidad, Autonomía personal y situaciones de Dependencia (EDAD), 2008. Inebase. Microdatos. Ficheros de sintaxis disponibles en: http://www.imsersomayores.csic.es/estadisticas/encuestas/index.html

Katz, S.; Akpom, A. (1976): "A measure of primary sociobiological functions". International Journal of Health Services, vol. 6, 3, p. 493-507.

Lafortune, G.; Balestat, G.; Disability Study Expert Group (2007): Trends in Severe Disability Among Elderly People. OCDE Health Working Papers, 26, 77 p. Disponible en: http://www.oecd.org/dataoecd/13/8/38343783.pdf

LEY 39/2006 de 14 de diciembre de Promoción de la Autonomía Personal y Atención a las Personas en Situación de Dependencia. Disponible en:

http://www.imsersomayores.csic.es/documentos/legislacion/normas/doc-3383.pdf 
Morán Aláez, E. (1999): "Estimación de la población dependiente y sus características sociodemográficas". En: Rodríguez Cabrero, G.: La protección social..., cap. 3. p. 111-161.

OMS (2001): CIF. Clasificación Internacional del Funcionamiento, de la Discapacidad y de la Salud. Madrid, Imserso, 320 p. Disponible en: http://www.imsersomayores.csic.es/documentos/documentos/oms-clasificacion01.pdf

Palacios Ramos, E.; Abellán García, A.; Esparza Catalán, C. (2008): Diferentes estimaciones de la discapacidad y dependencia en España. Informes Portal Mayores, 56, 10 p. Disponible en:

http://www.imsersomayores.csic.es/documentos/documentos/abellanestimaciones-11.pdf

Pérez Díaz, J. (2005): "Consecuencias sociales del envejecimiento demográfico". Papeles de Economía Española, 104, p. 210-226.

Puga González, M.D.; Abellán García, A. (2004): El proceso de discapacidad. F. Pfizer, $210 \mathrm{p}$.

Puga, M.D.; Abellán, A.; Sancho, M.T. (2006): "Mayores y familia en la sociedad actual". En: Fundación Encuentro: Informe España 2006. Madrid, 352 p.; p. 261333.

Puyol Antolín, R.; Abellán García, A. (Coord.) (2006): Envejecimiento y dependencia. Una mirada al panorama futuro de la población española. Madrid, Mondial Assistance, 234 p. Disponible en:

http://www.imsersomayores.csic.es/documentos/documentos/abellanenvejecimiento-01.pdf

Querejeta González, M. (2004): Discapacidad/dependencia. Unificación de criterios de valoración y clasificación. Madrid, Imserso, 162 p. Disponible en: http://www.imsersomayores.csic.es/documentos/documentos/querejetadiscapacidad-01.pdf

Rodríguez Cabrero, G. (Coord.) (1999): La protección social de la dependencia. Madrid, Imserso, 629 p.

Rodríguez Cabrero, G. (2004): Protección social de la dependencia en España. Madrid, Fundación Alternativas, Doc. 44/2004, 68 p. Disponible en:

http://www.imsersomayores.csic.es/documentos/documentos/rodriguezproteccion-02.pdf

Spector, W.D.; Fleishman, J.A. (1998): "Combining Activities of Daily Living with Instrumental Activities of Daily Living to Measure Functional Disability". Journal of Gerontology, Social Sciences. Vol. 53B, 1, p. S46-S57. 
Verbrugge, L.M.; Yang, L.S.; Juarez, L. (2004): "Severity, timing and structure of disability". Sozial- und Präventivmedizin/Social and Preventive Medicine, 49, p. 110-121.

Whiteneck, G. (2005): "Conceptual Models of Disability: Past, Present and Future". En: Institute of Medicine: Workshop on Disability in America, 275 p.

Wilkin, D. (1987): "Conceptual problems in dependency research". Social Science and Medicine, vol. 24, 10, p. 867-873. 